\title{
Precision engagement: the PMI's success will depend on more than genomes and big data
}

\author{
Jennifer K. Wagner, JD, PhD¹, Cathryn Peltz-Rauchman, PhD², Alanna Kulchak Rahm, PhD, LGC ${ }^{1}$ and \\ Christine C. Johnson, $\mathrm{PhD}, \mathrm{MPH}^{2}$
}

The debut of the Precision Medicine Initiative (PMI) is imminent. Insights from the Geisinger Health System (GHS) and the Henry Ford Health System (HFHS) suggest that realization of the PMI's promised potential to advance biomedical discoveries and improve the health of the American people will be determined less by the supposed stars of the show (i.e., genomics and big data) and more by its supporting cast (i.e., the eager, engaged patient-participants). Nuanced perspectives underscore the importance of precision engagement to understand context, meet prospective patient-participants where they are, and optimize and sustain each individual's willingness and ability to participate actively in PMI research activities.

Researchers at GHS and HFHS, both with extensive biobank and patient-engagement experience, initiated independent efforts in early 2016 to understand patients' perspectives regarding the upcoming PMI. GHS, an integrated health system in central and northeast Pennsylvania known for innovation as a learning health-care system and serving rural and underserved populations, hosted a series of open discussion forums and administered an online survey to a random sample of 10,000 patient-participants from its MyCode Community Health Initiative, which comprises more than 100,000 patientparticipants. HFHS, an integrated health system in metropolitan Detroit, Michigan, that is known for its population science research and serves an urban/suburban and diverse population, administered an online survey to the Henry Ford Insights Community, a virtual patient community of 4,300 patient-participants who regularly provide opinions and ideas used to improve health-care experiences. Both GHS and HFHS collected responses over a 2 -week period. GHS received 566 responses (6\% response rate) and HFHS received 1,576 (37\%). Together, the results of these independent surveys provide insight regarding the importance of engagement for the PMI's success and can be compared with results reported from a national online survey administered for the National Institutes of Health in $2015 .^{1}$

The PMI, which presupposes increased connectivity and broad interest of the American people in being active research participants or "partners," intends to recruit a cohort representative of the US population at an unprecedented scalea novel approach for which not every detail can be controlled and not every potential pitfall can be anticipated. ${ }^{2}$ The PMI is a "comprehensive effort" to explore health outcomes beyond those measurable in a clinic. ${ }^{3}$ It will involve public and private partnerships working together to advance genomic medicine ${ }^{4}$ and will depend heavily on understanding and supporting engaged patients. ${ }^{5}$ Patient perspectives gleaned from these surveys (summarized in Tables 1 and 2) and GHS and HFHS experiences with other deliberative engagement (summarized in Table 3) provide insights for the PMI. Significant investment and attention must be directed to engagement in order to overcome the tendency for large collaborative projects to impose a one-size-fits-all approach to the planned communications with, recruitment of, and interactions with the prospective members of the PMI cohort.

Setting realistic expectations for the PMI and its prospective participants is critical, particularly regarding what information will be shared with participants, when and how it will be shared, and how to interpret and influence varying rates of participation once they are enrolled. Response rates for various activities will be highly dependent on the framing of the PMI during recruitment (e.g., whether passive participation would be permitted; whether recruitment and retention quotas set for healthcare provider organizations (HPOs) lead to the imposition of minimum participation thresholds that participants must meet to remain in the PMI cohort; or whether patients have the ability to moderate their own levels of minimal, moderate, or maximum involvement) and resources devoted to maximizing involvement (e.g., use of reminders, prompts, and targeted incentives).

Patients have indicated that learning something about their health is a main motivator for participating in the PMI, but the logistics and the scope of content have yet to be determined. Managing the PMI cohort and keeping participants interested if the processing pipeline is slow, complex, or not communicated will be difficult. Even the decision regarding who (a coordinating center or a local HPO) will serve as the point of contact for each participant is significant. The courage and energy required

${ }^{1}$ Geisinger Health System, Danville, Pennsylvania, USA; ${ }^{2}$ Henry Ford Health System, Detroit, Michigan, USA. Correspondence: Jennifer K. Wagner (jwagner1@geisinger.edu) 
Table 1 Summary of Geisinger Health System survey findings

\section{Survey characteristics and findings}

Target population
Sampled population
Sample size
Key finding 1: interest in joining the PMI
Key finding 2: willingness to contribute data from diverse sources

Key finding 3: willingness to travel for baseline examination or sample collection

97,000 adults then enrolled in a MyCode

Random sample of 10,000

$N=565$

44.7\%: "I am eager to participate"

47.7\%: "I might be interested in this new PMI study, but I want more information"

7.6\%: "I am not interested...MyCode is enough for me"

$64.4 \%$ : Surveys on paper

$91.5 \%$ : Surveys online

$51.3 \%$ : Surveys via mobile apps

$61.1 \%$ : Wearables

$62.1 \%$ : Home health monitoring devices

$63.1 \%$ : Environmental monitoring devices

$38.5 \%:<10$ miles from home

$75.3 \%$ would not travel $>20$ miles from their home for the examination

36.8\%: 10-20 miles from home

$13.4 \%: 21-30$ miles from home

$5.03 \%$ : 31-40 miles from home

$6.30 \%$ : >40 miles from home

Key finding 4: preference for when/where the examination occurs (top ranked choice shown)

81.0\%: "At my local clinic on a day and time I choose and schedule in advance"

9.13\%: "At my home on a day at a time I choose and schedule in advance"

4.82\%: "At regularly scheduled community events that take place only in specific locations within the Geisinger Health System"

3.55\%: "At a local pharmacy or walk-in clinic (such as CareWorks, CVS, or Walgreens) whenever I choose"

$0.50 \%$ : "At regularly scheduled community events in my local area (like a community blood drive)"

Key finding 5: most convenient time and days for research activities (top-ranked choice shown)

43.4\%: Mornings during the week

$22.2 \%$ : Afternoons during the week

$14.8 \%$ : Evenings during the week

$12.2 \%$ : Mornings during the weekend

$4.08 \%$ : Afternoons during the weekend

$2.04 \%$ : Evenings during the weekend

Key finding 6: concerns about the PMI

55.3\%: "I do not have any concerns about this new PMI study"

28.1\%: "I am worried about who could have access to my information"

17.1\%: "I do not know enough about (or might not approve of) the research that would use my information if I participated in this new PMI study"

15.3\%: "I am worried I will not be able to do everything involved"

4.68\%: "I would not want to have a physical examination"

$3.64 \%$ : "I do not trust the $[\mathrm{NIH}]$ with my information and samples"

$1.30 \%$ : "My family members might not want me to participate"

Key finding 7: reasons to participate
86.2\%: "I think it is important to help researchers improve our understanding of genetics and disease risk, treatment, and prevention"

81.0\%: "I think it is important to contribute to future research"

$56.3 \%$ : "I think I could benefit from this type of research"

48.1\%: "I think my family members could benefit from this type of research"

31.6\%: "I feel a moral duty to society to participate in research"

$22.7 \%$ : "I think it is fun to participate in research"

$1.73 \%$ : "None of these reasons apply to me"

$\mathrm{NIH}$, National Institutes of Health; PMI, Precision Medicine Initiative. 
Table 2 Summary of Henry Ford Health System survey findings

Survey characteristics and findings (total respondent \%

(African-American Respondent \%))

Target population

Sampled population

Sample size

Key finding 1: likelihood of recruitment by different sources

Key finding 2: likelihood of recruitment by different methods

Key finding 3: importance of knowing details of PMI before agreeing to participate

Key finding 4: benefits and barriers to participating in the PMI

Key finding 5: preference for where the baseline examination occurs

\author{
Current HFHS patients \\ HFHS insights community $(n=4,300)$ \\ $N=1,576(13 \% \mathrm{AA})$ \\ $\%$ Likely/very likely to participate if asked by the following: \\ 76\% (72\% AA): Your physician \\ 57\% (46\% AA): A family member \\ $55 \%(61 \% A A):$ A health-care system \\ $52 \%$ (55\% AA): A nurse at your physician's office \\ $43 \%$ (54\% AA): Your health insurance provider \\ $21 \%(33 \% \mathrm{AA})$ : A church group
}

$\%$ Likely/very likely to participate if recruited through the following methods:

$59 \%(61 \% A A)$ : Asked in person

$57 \%$ (64\% AA): Received a message through MyChart (patient portal)

$41 \%$ (47\% AA): Received a mailed letter

$41 \%(55 \% \mathrm{AA})$ : Invited to a local event to learn more

$18 \%$ (27\% AA): Received a phone call

$8 \%(14 \%$ AA): Saw a post on social media

$\%$ Indicated the following were important/very important:

94\% (95\% AA): What information and laboratory work would be needed

$91 \%(93 \% A A)$ : Where/how the information and laboratory results would be obtained and stored

94\% (96\% AA): Who has access to my health information

$94 \%(97 \%$ AA): Security measures to ensure my information is secure

$90 \%(90 \% A A)$ : Details of research studies

93\% (94\% AA): Results from research studies

$\%$ Benefit:

$\%$ Barrier:

$92 \%(88 \% \mathrm{AA})$ : I may find out new health

information about myself

$42 \%(46 \%$ AA): I may not know study details at the time when my information is collected

$85 \%(86 \% \mathrm{AA})$ : I will be part of studies that can help keep people healthy and develop $16 \%$ (23\% AA): No financial incentives for participating new treatments

$33 \%$ (33\% AA): My whole family can

participate

Which of the following locations would you be comfortable with?

$76 \%$ (66\% AA): Your primary care

physician's office

$78 \%$ (75\% AA): HFHS facility close to your

home

$18 \%(14 \%$ AA): Non-HFHS facility close to

your house

$3 \%(4 \%$ AA): Health fair in a local

community

Key finding 6: benefits and barriers to the baseline exam

\section{$\%$ Benefit:}

$88 \%$ ( $87 \%$ AA): Examination could be performed as part of an existing

$\%$ Barrier:

appointment

$39 \%$ (34\% AA): A separate appointment would be needed

$82 \%$ (79\% AA): Examination locations would be $<10$ minutes from your home $65 \%(72 \% \mathrm{AA})$ : Examinations would be available on a walk-in basis

Key finding 7: willingness to participate long-term

$14 \%(19 \% A A)$ : Would not be willing to participate long-term

AA, African-American; HFHS, Henry Ford Health System; PMI, Precision Medicine Initiative. 
Table 3 Examples of findings from various deliberative patient-engagement activities Key finding

Key finding 1: balancing priorities and motivators for the PMI

When discussing features that might increase participation rates, participants prioritize several features above being included as partners in the research design and process or having opportunities to learn how research will be conducted. Features prioritized, in rank order, were periodic updates about the study's progress, access to copies of data collected and individual research results, and an ability to recruit friends and family members to participate in study activities along with them.

Key finding 2: creating cohort strategies

Participants supported cohort strategies such as "Senior Siblings" (siblings older than 65 who could participate together). Participants wanted gatherings, i.e., opportunities to socialize and "do" research with others (including friends and family); however, when annual family reunions were suggested as a possible time to do this, participants said that they do not get to see family often enough and this would not be how they would want to use the precious family time they do get to enjoy. Alternative suggestions included church and community fundraiser-style events and co-worker activities. Participants wanted to feel connected to others throughout the PMI cohort and suggested use of webinars.

Key finding 3: ideas to promote involvement and retention

Participants identified several features that would make PMI "more fun, interesting, and interactive," including a research idea incubator, accelerator, or "Shark Tank"-like program; an online or mobile research dashboard to learn the status of their own specimens and data in studies and research progress; and social components (e.g., "meet-ups").

Key finding 4: input on research design, questions, and methods

Participants want the PMI to study the health impact of environmental exposures (suggesting several research priorities, e.g., water from nearby fracking activities, effects of pesticides and herbicides in the food chain, and air quality related to automobile emissions and materials); however, when discussing the inclusion of microbiome studies, participants were divided (with participants remarking that swabbing items "is just too much," emphasizing a need for "edutainment" about microbiome studies before data collection, and expressing that willingness to contribute would vary depending on whether data could be automatically transferred from monitoring devices placed in the home/office rather than requiring manual submission). Participants preferred iOS and Android devices over Windows-based ones and expressed interest in continuing to use wearables and other devices they already own.

Key finding 5: depositing research results in the EMR

Adviser input about having research data in EMR included queries as to (i) whether research results could be used clinically to avoid repeating tests and (ii) whether patients who drop out will be treated differently by providers if the research EMR is retained.

Key finding 6: obtaining laboratory or test data in the patient portal immediately when available

The majority of patients felt confident about their ability to access their health information, saying it helps them to prepare for discussions with their health-care provider.

Key finding 7: importance of incorporating patient input at the beginning of the research design phase

Patient advisers said they would not participate in a proposed international, randomized, clinical trial because they perceived one treatment as being superior to the others. The advisers also proposed helpful modifications to a mobile app developed for cancer patients, but by the time they were asked, the app could not be changed.

\section{Source of finding}

Open-forum discussions held at Geisinger

(January 2016) with enrolled MyCode participants

Open-forum discussions held at Geisinger

(January 2016) with enrolled MyCode participants
Open-forum discussions held at Geisinger

(January 2016) with enrolled MyCode participants

Open-forum discussions held at Geisinger

(January 2016) with enrolled MyCode participants

EMR, electronic medical record; PCORI P2P, Patient-Centered Outcomes Research Institute Pipeline to Proposal; PMI, Precision Medicine Initiative.

to initiate communication are more burdensome to participants if the contact is unfamiliar. A centralized rather than local primary contact raises the possibility that sources of confusion or bad experiences with PMI will go unnoticed, unmeasured, and unaddressed as well as the possibility that individuals with questions or concerns will find it easier to stay silent or back out entirely rather than obtain clarification. Centralized messaging from the coordinating center to the emerging cohort in the earliest phase might not be as effective as orchestration by the coordinating center of a set of carefully tailored, flexible approaches to inspire eager, active participation in which the specific HPOs initiate recruitment through the existing, trusted relationships with the patients and communities they serve and leverage prior knowledge of patient and community preferences, needs, and interests. Both surveys revealed general preferences that baseline examinations be performed in a local HPO facility. The flexibility that will be required for successful long-term commitment and involvement is not only that which addresses variation between patient-participants but also that which accommodates the variation foreseeable for each patient-participant's interest, motivation, and ability to engage over time.

There is a fine line between motivating and alienating participants, and a fine line between, on the one hand, the need 
to avoid missing data and maximize sample size and statistical power and, on the other hand, an individual participant's autonomous decision to decline some activities while remaining an active member of the PMI cohort. Accommodating life interruptions (such as needing to take a second job, caring for a new baby, or getting arrested) that might cause an individual's involvement in research activities to plummet and leveraging a person's heightened interest in research related to a particular event (such as a friend's diagnosis with a condition prompting an increased interest in a person's contribution of data, time, energy, and money to research being conducted for that disease) are important considerations. Such occurrences are likely to vary in frequency and magnitude between demographic groups.

Navigating the nuanced perspectives of patients and communities, such as those learned by GHS and HFHS in their extensive and ongoing patient-engagement experience, requires skill and finesse not easily transferred between entities or newcomers. For example, there is a strong desire for a social and emotional bond (and actual interactions and communication) between members of the PMI cohort. However, this desired communitas should not be confused with intimacy. Although the ability of family members to participate is seen as a benefit, and the potential to learn about one's genetic ancestry might motivate individuals to join the PMI cohort, approaching extended families at annual reunions to participate in research activities could be received as an unwelcome intrusion. Additionally, not everyone who is interested in participating has the ability to participate in all PMI activities. This suggests a recruitment barrier and an engagement challenge-although individuals might be enrolled in the PMI and willing to contribute data from diverse sources, they might lack the necessary resources (such as a reliable Internet connection or cell signal at home needed to sync devices or submit online surveys) to contribute fully. Potential participants might want to know the full extent of activities expected of them before agreeing to join the PMI cohort. Accordingly, it would be advantageous to determine whether patient-partners have an option, at the outset, to participate at different levels of intensity.

Initial PMI plans suggest that it will include deliberative involvement of patients as partners in research (helping to develop research priorities, select methods, conduct the research, and distribute the results). Such types of engagement require considerable infrastructure and institutional commitment to support and manage. Public messaging of partnerships with patients and the public without corresponding actions could convert early champions into critics. Ongoing diligence is required to optimize engagement and implement participant-generated and -supported research priorities for PMI. Two such areas of ongoing attention for HFHS and GHS are (i) the onboarding processes for patients in various research roles (e.g., screening, matching, and training not only for patients but also for research personnel to enable a scientifically robust, ethically sound, productive, and meaningful collaboration to occur for all parties) and (ii) solicitation of patient input regarding which environmental factors, medical conditions, or health outcomes are prioritized for study.
Genomics and big data, cast as the leads, continue to bask in the PMI's spotlight; however, they can only be as strong as their supporting cast. Without patients' eagerness to participate in PMI activities, willingness to contribute biospecimens and data from diverse sources, long-term commitment, and contentment with the pace of progress, the performance of PMI genomics and big data will underachieve. We must continue to emphasize the essential role that patients-involved in PMI through precision engagement-play in realizing the potential greatness of precision medicine. Precision engagement requires significant resources and dedication to meet patients where they are (spatially, temporally, psychologically, socially, economically, etc.) and to implement strategies for research activities, recruitment, and retention that appeal to people with diverse needs, interests, and capacities for research participation; leverage the diverse motivations patients have for participating; foster communitas; and adequately accommodate life's interruptions and distractions that could limit involvement despite patientparticipants' intentions.

\section{ACKNOWLEDGMENTS}

The research protocol at Geisinger Health System (GHS) (20160106) was approved on 8 January 2016 by the Mycode Governing Board and by the Geisinger institutional review board as category 2 exempt research. All participants gave implied consent via submission of survey responses. At Henry Ford Health System (HFHS), this research is a part of the AHRQ-funded (R24 HS022417) Patient-Centered Outcomes Research Center infrastructure and was approved by the HFHS institutional review board (8288) with an expedited review. All participants gave implied consent by responding electronically to an e-mailed survey request.

The GHS authors thank the patients in MyCode for their participation, as well as Daniel Davis, Carroll Flansburg, Sharon Larson, and Andrew Faucett for input regarding the development of the GHS surveys. The HFHS authors thank the patients in the HFHS Insights Community and the Patient Engaged Research Center staff for input regarding the development of the HFHS surveys.

After acceptance of this manuscript, GHS and HFHS were each issued awards to be a part of the PMI Cohort Program (NIH Award \#1OT2OD024609-01 and \#10T2OD024610-01, respectively).

\section{DISCLOSURE}

The authors declare no conflict of interest.

\section{REFERENCES}

1. Kaufman DJ, Baker R, Milner LC, Devaney S, Hudson KL. A survey of U.S. adults' opinions about conduct of a Nationwide Precision Medicine Initiative $₫$ cohort study of genes and environment. PLoS One 2016;11:e0160461.

2. Collins FS, Varmus H. A new initiative on precision medicine. $N$ Engl J Med 2015:372:793-795.

3. Riley WT, Nilsen WJ, Manolio TA, Masys DR, Lauer M. News from the NIH: potential contributions of the behavioral and social sciences to the precision medicine initiative. Trans/ Behav Med 2015;5:243-246.

4. Ashley EA. The precision medicine initiative: a new national effort. JAMA 2015;313:2119-2120.

5. Anderson M, Kimberly McCleary K. On the path to a science of patient input. Sci Trans/ Med 2016:8:336ps11. 